\title{
Postmarket medical device safety: moving beyond voluntary reporting
}

\author{
Frederic S Resnic, Arjun Majithia
}

Cardiovascular Medicine, Lahey Hospital and Medical Center and Tufts University School of Medicine, Burlington, MA, USA

\section{Correspondence to}

Dr Frederic S Resnic, Lahey Hospital and Medical Center, Tufts University School of Medicine;

frederic.resnic@lahey.org

Accepted 25 September 2017 Published Online First 9 October 2017

\section{SLinked}

http://dx.doi.org/10.1136/ bmjqs-2017-006481

Check for updates

To cite: Resnic FS, Majithia A. BMJ Qual Saf

2018:27:174-175.
Assuring public safety after widespread adoption of medical devices is critically important, and has been identified as a priority by the Food and Drug Administration (FDA). ${ }^{1}$ Growing public concern regarding postmarket safety of medical devices stems in part from an increasing recognition that medical device failure, although infrequent, may lead to injury. Regulatory agencies, such as FDA, are faced with the competing pressures of ensuring patient safety while simultaneously improving the efficiency of medical device approval. Unfortunately, premarket medical device approval studies are frequently limited by small sample size, restricted patient and provider populations, and inadequate duration of follow-up. ${ }^{2}{ }^{3}$ Therefore, strengthening approaches of postmarket safety evaluation are increasingly important.

The FDA strategy for postmarket safety evaluation has relied extensively on reporting of adverse events by physicians, patients and device manufacturers through the Medical Device Reporting system. It has become increasingly apparent that, despite a vested interested in device performance, physicians, hospitals and patients significantly under-report adverse events, with a US Inspector General report estimating that only $0.5 \%$ of all device-related adverse events were reported to FDA. ${ }^{2}$

In BMJ Quality and Safety, Gagliardi and colleagues ${ }^{4}$ report the results of their investigation of the factors that influence under-reporting of medical device adverse events. The authors conducted detailed and structured interviews with 22 physicians who implant cardiovascular and orthopaedic medical devices to determine which factors influenced non-reporting behaviour. All of the participants interviewed believed that adverse device event reporting was either unnecessary, not possible or futile. Providers cited lack of feedback when reporting events, and cumbersome reporting systems as factors contributing to non-reporting. This study further supports the observation that voluntary reporting frequently results in incomplete ascertainment of realworld device safety outcome data and an inability to reliably estimate adverse event rates.

Beyond passive reporting of adverse medical device events, additional strategies to support postmarket evaluation by FDA have included postapproval studies (PAS). However, both the effectiveness and value of PAS have been questioned. In an analysis of 223 studies mandated by FDA between 2005 and 2011, there were no instances of penalties or fines associated with delays in implementing PAS and no such study led to withdrawal or medical device recall. ${ }^{5}$ Additionally, PAS are costly. A separate analysis of 277 FDA-required PAS between 2005 and 2013 estimated a median cost of $\$ 2.16$ million per study, and $\$ 1.22$ billion in total costs. ${ }^{6}$ While it might appear that the financial burden of PAS is absorbed by industry, these costs are ultimately distributed to the healthcare system and onto patients through device costs.

Recognising the need to modernise the systems in place to assure postapproval safety of medical devices, FDA has articulated strategic priorities which include promoting and developing representative clinical medical device registries and development of new methods for evidence evaluation, including active surveillance of these clinical data sources. ${ }^{1}$ We believe that strategies that can leverage complementary large and detailed clinical data sources will offer tremendous value in performing more routine and thorough postmarket assessments and to provide more accurate safety estimates. For example, linking detailed device-specific registry data with longitudinal health 
outcomes captured in electronic health records could allow more complete assessment of postmarket safety. Given the lack of evidence to support the effectiveness of current FDA-mandated postmarket studies, and their associated high costs, we recommend consideration of reallocation of resources towards other strategies for safety evaluation, including the development of national device registries and the routine use of prospective, active surveillance of these data sources. Such an approach should be designed to incorporate the best available statistical tools to study medical devices in as near real time as feasible, in order to identify potential safety concerns early in their widespread use as well as potentially identify opportunities to use these innovative therapies in patients who may not have previously been studied. In fact, this approach of active safety surveillance has already been shown to be feasible, and has been used to prospectively identify relative safety differences of commonly used devices in a national cardiovascular registry. ${ }^{7}$

We remain optimistic that utilising such complementary approaches towards strengthening postmarket safety evaluation will allow regulatory agencies throughout the world to strike an appropriate balance between ensuring public safety, while continuing to support innovation. Such a strategy will also help to address some of the inherent limitations of a passive adverse event tracking system highlighted by Gagliardi and colleagues.
Competing interests None declared.

Provenance and peer review Commissioned; internally peer reviewed.

(C) Article author(s) (or their employer(s) unless otherwise stated in the text of the article) 2018. All rights reserved. No commercial use is permitted unless otherwise expressly granted.

\section{REFERENCES}

1 Administration UFaD. Strengthening our national system for postmarket device surveillance, 2012.

2 Resnic FS, Normand SL. Postmarketing surveillance of medical devices-filling in the gaps. N Engl J Med 2012;366:875-7.

3 Normand SL, Hatfield L, Drozda J, et al. Postmarket surveillance for medical devices: America's new strategy. BMJ 2012;345:e6848.

4 Gagliardi AR, Ducey A, Lehoux P, et al. Factors influencing the reporting of adverse medical device events: qualitative interviews with physicians about higher risk implantable devices. BMJ Qual Saf 2018;27:190-8.

5 Reynolds IS, Rising JP, Coukell AJ, et al. Assessing the safety and effectiveness of devices after US Food and Drug Administration approval: FDA-mandated postapproval studies. JAMA Intern Med 2014;174:1773-9.

6 Wimmer NJ, Robbins S, Ssemaganda $\mathrm{H}$, et al. Assessing the cost burden of United States FDA-mandated post-approval studies for medical devices. J Health Care Finance 2016.

7 Resnic FS, Majithia A, Marinac-Dabic D, et al. Registry-Based Prospective, Active Surveillance of Medical-Device Safety. N Engl J Med 2017;376:526-35. 